\title{
Effect of electron-electron interaction on the phonon-mediated spin relaxation in quantum dots
}

\author{
Juan I. Climente, ${ }^{1, *}$ Andrea Bertoni, ${ }^{1}$ Guido Goldoni, ${ }^{1,2}$ Massimo Rontani, ${ }^{1}$ and Elisa Molinari ${ }^{1,2}$ \\ ${ }^{1}$ CNR-INFM National Center on NanoStructures and BioSystems at Surfaces (S3), Via Campi 213/A, 41100 Modena, Italy \\ ${ }^{2}$ Dipartimento di Fisica, Università degli Studi di Modena e Reggio Emilia, Via Campi 213/A, 41100 Modena, Italy
}

(Received 5 April 2007; revised manuscript received 6 June 2007; published 6 August 2007)

\begin{abstract}
We estimate the spin relaxation rate due to spin-orbit coupling and acoustic phonon scattering in weakly confined quantum dots with up to five interacting electrons. The full configuration interaction approach is used to account for the interelectron repulsion, and Rashba and Dresselhaus spin-orbit couplings are exactly diagonalized. We show that electron-electron interaction strongly affects spin-orbit admixture in the sample. Consequently, relaxation rates strongly depend on the number of carriers confined in the dot. We identify the mechanisms which may lead to improved spin stability in few electron $(>2)$ quantum dots as compared to the usual one and two electron devices. Finally, we discuss recent experiments on triplet-singlet transitions in GaAs dots subject to external magnetic fields. Our simulations are in good agreement with the experimental findings, and support the interpretation of the observed spin relaxation as being due to spin-orbit coupling assisted by acoustic phonon emission.
\end{abstract}

DOI: 10.1103/PhysRevB.76.085305 PACS number(s): 73.21.La, 71.70.Ej, 72.10.Di, 73.22.Lp

\section{INTRODUCTION}

There is currently interest in manipulating electron spins in quantum dots (QDs) for quantum information and quantum computing purposes. ${ }^{1-3} \mathrm{~A}$ major goal in this research line is to optimize the spin relaxation time $\left(T_{1}\right)$, which sets the upper limit of the spin coherence time $\left(T_{2}\right): T_{2} \leqslant 2 T_{1} \cdot{ }^{4}$ Therefore, designing two-level spin systems with long spin relaxation times is an important step towards the realization of coherent quantum operations and read-out measurements. Up to date, spin relaxation has been investigated almost exclusively in single-electron ${ }^{4-19}$ and two-electron ${ }^{20-30}$ QDs. Spin relaxation in QDs with a larger number of electrons has seldom been considered, ${ }^{28,31}$ even though Coulomb blockade makes it possible to control the exact number of carriers confined in a QD. ${ }^{32}$ Yet, recent theoretical works suggest that Coulomb interaction renders few-electron charge degrees of freedom more stable than single-electron ones, ${ }^{33}$ which leads to the question of whether similar findings hold for spin degrees of freedom. Moreover, in weakly confined QDs, acoustic phonon emission assisted by spin-orbit (SO) interaction has been identified as the dominant spin relaxation mechanism when cotunneling and nuclei-mediated relaxation are reduced. ${ }^{6,8,31}$ The combined effect of Coulomb interaction and SO coupling has been shown to influence the energy spectrum of few-electron QDs profoundly, ${ }^{34-36}$ but the consequences on the spin relaxation remain largely unexplored. ${ }^{37}$

In Ref. 28 we investigated the effect of a magnetic field on the triplet-singlet (TS) spin relaxation in two and fourelectron QDs with SO coupling, so as to understand related experimental works. Motivated by the very different response observed for different number of confined particles, in this work we shall focus on the role of electron-electron interaction in spin relaxation processes, extending our analysis to different number of carriers, highlighting, in particular, the different physics involved in even and odd number of confined electrons. Furthermore, we will explicitly compare the predictions of our theoretical model with very recent ex- periments on spin relaxation in two-electron GaAs QDs. ${ }^{29}$

We study theoretically the energy structure and spin relaxation of $N$ interacting electrons $(N=1-5)$ in parabolic GaAs QDs with SO coupling, subject to axial magnetic fields. Both Rashba $^{38}$ and Dresselhaus ${ }^{39}$ SO terms are considered, and the electron-electron repulsion is accounted for via the full configuration interaction method. ${ }^{40,41}$ By focusing on the two lowest spin states, two different classes of systems are distinguished. For $N$ odd $(1,3,5)$ and weak magnetic fields, the ground state is a doublet and then the two-level system is defined by the Zeeman-split sublevels of the lowest orbital. For $N$ even $(2,4)$, the two-level system is defined by a singlet and a triplet. We analyze these two classes of systems separately because, as we shall comment below, the physics involved in the spin transition differs. Thus, we compare the phonon-induced spin relaxation of $N=1,3,5$ electrons and that of $N=2,4$ separately. As a general rule, the larger the number of confined carriers, the stronger the SO mixing, owing to the increasing density of electronic states. This would normally yield faster relaxation rates. However, we note that this is not necessarily the case, and few-electron states may display comparable or even slower relaxation than their single-electron and two-electron counterparts. This is due to characteristic features of the few-particle energy spectra which tend to weaken the admixture between the initial and final spin states. In $N$-odd systems, it is the presence of low-energy quadruplets for $N>1$ that reduces the admixture between the Zeeman sublevels of the (doublet) ground state, hence inhibiting the spin flipping. In $N$-even systems, electronic correlations partially quench phonon emission, ${ }^{33}$ and the relaxation can be further suppressed for $N>2$ if one selects initial and final spin states differing in more than one quantum of angular momentum, which inhibits direct triplet-singlet SO mixing via linear Rashba and Dresselhaus SO terms. ${ }^{28}$ Noteworthy, all these effects are connected with Coulomb interaction between confined carriers.

The paper is organized as follows. In Sec. II we give details about the theoretical model we use. In Sec. III we 
study the energy structure and spin relaxation of a QD with an odd number of electrons $(N=1,3,5)$. In Sec. IV we do the same for QDs with an even number of electrons $(N=2,4)$. In Sec. V we compare our numerical simulations with experimental data recently reported for $N=2 \mathrm{GaAs}$ QDs. Finally, in Sec. VI we present the conclusions of this work.

\section{THEORY}

We consider weakly confined GaAs/AlGaAs QDs, which are the kind of samples usually fabricated by different groups to investigate spin relaxation processes. ${ }^{7,8,20,22}$ In these structures, the dot and the surrounding barrier have similar elastic properties, and the lateral confinement (which we approximate as circular) is much weaker than the vertical one. A number of useful approximations can be made for such QDs. First, since the weak lateral confinement gives interlevel spacings within the range of few $\mathrm{meV}$, only acoustic phonons have significant interaction with bound carriers, while optical phonons can be safely neglected. Second, the elastically homogeneous materials are not expected to induce phonon confinement, which allows us to consider three-dimensional bulk phonons. Finally, the different energy scales of vertical and lateral electronic confinement allow us to decouple vertical and lateral motion in the building of single-electron spin orbitals. Thus, we take a parabolic confinement profile in the in-plane $(x, y)$ direction, with single-particle energy gaps $\hbar \omega_{0}$, which yields the Fock-Darwin states. ${ }^{42}$ In the vertical direction $(z)$ the confinement is provided by a rectangular quantum well of width $L_{z}$ and height determined by the band offset between the QD and barrier materials (the zero of energy is then the bottom of the conduction band). The quantum well eigenstates are derived numerically. In cylindrical coordinates, the single-electron spin orbitals can be written as

$$
\psi_{\mu}\left(\rho, \theta, z ; s_{z}\right)=\frac{1}{\sqrt{2 \pi}} e^{i m \theta} R_{n, m}(\rho) \xi_{0}(z) \chi_{s_{z}}
$$

where $\xi_{0}$ is the lowest eigenstate of the quantum well, $\chi_{s_{z}}$ is the spinor eigenvector of the spin $z$ component with eigenvalue $s_{z}$, and $R_{n, m}$ is the $n$th Fock-Darwin orbital with azimuthal angular momentum $m$,

$$
R_{n, m}(\rho)=\frac{1}{l_{0}} \sqrt{\frac{n !}{(n+|m|) !}}\left(\frac{\rho}{l_{0}}\right)^{|m|} e^{-\rho^{2} / 2 l_{0}^{2}} \mathcal{L}_{n}^{|m|}\left(\frac{\rho^{2}}{l_{0}^{2}}\right) .
$$

In the above expression $\mathcal{L}_{n}^{|m|}$ denotes a generalized Laguerre polynomial and $l_{0}=\sqrt{\hbar / m^{*}} \omega_{0}$ is the effective length scale, with $m^{*}$ standing for the electron effective mass. The energy of the single-particle Fock-Darwin states is given by $E_{n, m}$ $=(2 n+1+|m|) \hbar \Omega_{c}+\frac{m}{2} \hbar \omega_{c}$, where $\omega_{c}=e B / m^{*} c$ is the cyclotron frequency and $\Omega_{c}=\sqrt{\omega_{0}^{2}+\left(\omega_{c} / 2\right)^{2}}$ is the total (spatial plus magnetic) confinement frequency.

With regard to Coulomb interaction, we need to go beyond mean field approximations in order to properly include electronic correlations, which play an important role in determining the phonon-induced electron scattering rate. ${ }^{43}$ Moreover, since we are interested in the relaxation time of excited states, we need to know both ground and excited states with comparable accuracy. Our method of choice is the full configuration interaction approach: the few-electron wave functions are written as linear combinations $\left|\Psi_{a}\right\rangle$ $=\Sigma_{i} c_{a i}\left|\Phi_{i}\right\rangle$, where the Slater determinants $\left|\Phi_{i}\right\rangle=\Pi_{\mu_{i}} c_{\mu_{i}}^{\dagger}|0\rangle$ are obtained by filling in the single-electron spin orbitals $\mu$ with the $N$ electrons in all possible ways consistent with symmetry requirements; here $c_{\mu}^{\dagger}$ creates an electron in the level $\mu$. The fully interacting Hamiltonian is numerically diagonalized, exploiting orbital and spin symmetries. ${ }^{40,41}$ The fewelectron states can then be labeled by the total azimuthal angular momentum $M=0, \pm 1, \pm 2, \ldots$, total spin $S$ and its $z$-projection $S_{z}$.

The inclusion of SO terms is done following a similar scheme to that of Ref. 44, although here we consider not only Rashba, but also linear Dresselhaus terms. For a quantum well grown along the [001] direction, these terms $\operatorname{read}^{38,39}$

$$
\begin{gathered}
\mathcal{H}^{R}=\frac{\alpha}{\hbar}\left(k_{y} s_{x}-k_{x} s_{y}\right), \\
\mathcal{H}^{D}=\gamma_{c}\left\langle k_{z}^{2}\right\rangle\left(k_{y} s_{y}-k_{x} s_{x}\right),
\end{gathered}
$$

where $\alpha$ and $\gamma_{c}$ are coupling constants, while $s_{j}$ and $k_{j}$ are the $j$ th Cartesian projections of the electron spin and canonical momentum, respectively, along the main crystallographic axes $\left[\left\langle k_{z}^{2}\right\rangle=\left(\pi / L_{z}\right)^{2}\right.$ for the lowest eigenstate of the quantum well]. The momentum operator includes a magnetic field $B$ applied along the vertical direction $z$. Other SO terms may also be present in the conduction band of a QD, such as the contribution arising from the system inversion asymmetry in the lateral dimension or the cubic Dresselhaus term. However, in GaAs QDs with strong vertical confinement, $\mathcal{H}^{R}$ and $\mathcal{H}^{D}$ account for most of the SO interaction. ${ }^{36}$

We rewrite Eqs. (3) and (4) in terms of ladder operators as

$$
\begin{aligned}
& \mathcal{H}^{R}=\frac{\alpha}{i \hbar}\left(k^{+} s^{-}-k^{-} s^{+}\right), \\
& \mathcal{H}^{D}=\frac{\beta}{\hbar}\left(k^{+} s^{+}+k^{-} s^{-}\right),
\end{aligned}
$$

where $k^{ \pm}$and $s^{ \pm}$change $m$ and $s_{z}$ by one quantum, respectively, and $\beta=\gamma_{c}\left(\pi / L_{z}\right)^{2}$ is the Dresselhaus in-plane coupling constant. It is worth mentioning that when only Rashba (Dresselhaus) coupling is present, the total angular momentum $j=m+s_{z}\left(j=m-s_{z}\right)$ is conserved. However, in the general case, when both coupling terms are present and $\alpha \neq \beta$, all symmetries are broken. Still, SO interaction in a large-gap semiconductor such as GaAs is rather weak, and the lowlying states can be safely labeled by their approximate quantum numbers $\left(M, S, S_{z}\right)$, except in the vicinity of the level anticrossings. ${ }^{11,26,45}$ Since the few-electron $M$ and $S_{z}$ quantum numbers are given by the algebraic sum of the singleparticle states $m$ and $s_{z}$ quantum numbers, it is clear from Eqs. (5) and (6) that Rashba interaction mixes $\left(M, S_{z}\right)$ states with $\left(M \pm 1, S_{z} \mp 1\right)$ ones, while Dresselhaus interaction mixes $\left(M, S_{z}\right)$ with $\left(M \pm 1, S_{z} \pm 1\right)$. 
The SO terms of Eqs. (5) and (6) can be spanned on a basis of correlated few-electron states. ${ }^{46}$ The SO matrix elements are then given by sums of single-particle contributions of the form

$$
\begin{aligned}
\left\langle n^{\prime} m^{\prime} s_{z}^{\prime}\left|\mathcal{H}^{R}+\mathcal{H}^{D}\right| n m s_{z}\right\rangle= & C_{R}^{*} \mathcal{O}_{n^{\prime} m^{\prime} n m}^{+} \delta_{m^{\prime} m+1} \delta_{s_{z}^{\prime} s_{z}-1} \\
& +C_{R} \mathcal{O}_{n^{\prime} m^{\prime} n m}^{-} \delta_{m^{\prime} m-1} \delta_{s_{z}^{\prime} s_{z}+1} \\
& +C_{D}^{*} \mathcal{O}_{n^{\prime} m^{\prime} n m}^{+} \delta_{m^{\prime} m+1} \delta_{s_{z}^{\prime} s_{z}+1} \\
& +C_{D} \mathcal{O}_{n^{\prime} m^{\prime} n m}^{-} \delta_{m^{\prime} m-1} \delta_{s_{z}^{\prime} s_{z}-1} .
\end{aligned}
$$

Here $C_{R}=\alpha$ and $C_{D}=-i \beta$ are constants for the Rashba and Dresselhaus interaction, respectively, and $\mathcal{O}^{ \pm}$are the form factors

$$
\begin{gathered}
\mathcal{O}_{n^{\prime} m^{\prime} n m}^{-}=\frac{l_{0}}{2} \int_{0}^{\infty} d t R_{n^{\prime} m^{\prime}}(t)\left(2 \sqrt{t} \frac{\partial}{\partial t}+\frac{m}{\sqrt{t}}+\frac{B l_{0}^{2} \sqrt{t}}{2}\right) R_{n m}(t), \\
\mathcal{O}_{n^{\prime} m^{\prime} n m}^{+}=\frac{l_{0}}{2} \int_{0}^{\infty} d t R_{n^{\prime} m^{\prime}}(t)\left[2\left(\frac{\partial}{\partial t}\right)^{\dagger} \sqrt{t}+\frac{m^{\prime}}{\sqrt{t}}+\frac{B l_{0}^{2} \sqrt{t}}{2}\right] R_{n m}(t),
\end{gathered}
$$

with $t=\rho^{2} / l_{0}^{2}$. The above forms factors have analytical expressions which depend on the set of quantum numbers $\left\{n^{\prime} m^{\prime}, n m\right\}$. The resulting SO-coupled eigenvectors are then linear combinations of the correlated states, $\left|\Psi_{A}^{S O}\right\rangle$ $=\Sigma_{a} c_{A a}\left|\Psi_{a}\right\rangle$.

We assume zero temperature, which suffices to capture the main features of one-phonon processes. ${ }^{9,16}$ Indeed, it is one-phonon processes that account for most of the lowtemperature experimental observations in the SO coupling regime. . $^{2,8,8,28,29,31}$ We evaluate the relaxation rate between the initial (occupied) and final (empty) states of the SOcoupled few-electron state, $B$ and $A$, using the Fermi's "golden rule:"

$$
\begin{aligned}
\tau_{B \rightarrow A}^{-1}= & \frac{2 \pi}{\hbar} \sum_{\nu \mathbf{q}}\left|\sum_{a b} c_{B b}^{*} c_{A a} \sum_{i j} c_{b i}^{*} c_{a j}\left\langle\Phi_{i}\left|V_{\nu \mathbf{q}}\right| \Phi_{j}\right\rangle\right|^{2} \delta\left(E_{B}-E_{A}\right. \\
& \left.-\hbar \omega_{q}\right),
\end{aligned}
$$

where the electron states $\left|\Psi_{K}^{S O}\right\rangle(K=A, B)$ have been written explicitly as linear combinations of Slater determinants, $E_{K}$ stands for the $K$ electron state energy, and $\hbar \omega_{q}$ represents the phonon energy. $V_{\nu \mathbf{q}}$ is the interaction operator of an electron with an acoustic phonon of momentum $\mathbf{q}$ via the mechanism $\nu$, which can be either deformation potential or piezoelectric field interaction. Details about the electron-phonon interaction matrix elements can be found elsewhere. ${ }^{33}$

In this work we study $\mathrm{GaAs}_{\mathrm{Al}} / \mathrm{Al}_{0.3} \mathrm{Ga}_{0.7}$ As QDs, using the following material parameters: ${ }^{47}$ electron effective mass $m^{*}$ $=0.067$, band offset $V_{c}=243 \mathrm{meV}$, crystal density $d$ $=5310 \mathrm{~kg} / \mathrm{m}^{3}$, acoustic deformation potential constant $D$ $=8.6 \mathrm{eV}$, effective dielectric constant $\epsilon=12.9$, and piezoelectric constant $h_{14}=1.41 \times 10^{9} \mathrm{~V} / \mathrm{m}$. The Landé factor is $g=-0.44 .^{5}$ As for GaAs sound speed, we take $c_{\mathrm{LA}}=4.72$ $\times 10^{3} \mathrm{~m} / \mathrm{s}$ for longitudinal phonon modes and $c_{\mathrm{TA}}=3.34$ $\times 10^{3} \mathrm{~m} / \mathrm{s}$ for transversal modes. ${ }^{48}$ Unless otherwise stated, a lateral confinement of $\hbar \omega_{0}=4 \mathrm{meV}$ and a quantum well width of $L_{z}=10 \mathrm{~nm}$ are assumed for the QD under study, and a Dressehlaus coupling parameter $\gamma_{c}=25.5 \mathrm{eV} \AA^{3}$ is taken, ${ }^{49}$ so that $\beta \approx 25 \mathrm{meV} \AA$. The value of the Rashba coupling constant can be modulated externally, e.g., with external electric fields. Here we will investigate systems both with and without Rashba interaction. When present, we shall mostly consider $\alpha=50 \mathrm{meV} \AA$ to represent the case where Rashba effects prevail over Dresselhaus ones.

Few-body correlated states $\left(M, S, S_{z}\right)$ are obtained using a basis set composed by the Slater determinants (SDs) which result from all possible combinations of 42 single-electron spin orbitals (i.e., from the six lowest energy shells of the Fock-Darwin spectrum at $B=0$ ) filled with $N$ electrons. For $N=5$, this means that the basis rank may reach $\sim 2 \times 10^{5}$. The SO Hamiltonian is then diagonalized in a basis of up to 56 few-electron states, which grants a spin relaxation convergence error below $2 \%$. Since SO terms break the spin and angular momentum symmetries, the SO-coupled states $\left|\Psi_{K}^{S O}\right\rangle$ are described by a linear combination of SDs coming from different $\left(M, S, S_{z}\right)$ subspaces. Thus, for $N=5$, the states are described by up to $\sim 8.5 \times 10^{5} \mathrm{SDs}$. To evaluate the electronphonon interaction matrix elements, we note that only a small percentage of the huge number of possible pairs of SDs $\left(\sim 7 \times 10^{11}\right.$ for $\left.N=5\right)$ may give nonzero matrix elements, owing to spin-orbital orthogonalities. We scan all pairs of SDs and filter those which may give nonzero matrix elements writing the determinants in binary representation and using efficient bit-per-bit algorithms. ${ }^{40,41}$ The matrix elements of the remaining pairs $\left(\sim 2 \times 10^{6}\right.$ for $\left.N=5\right)$ are evaluated using massive parallel computation.

\section{SPIN RELAXATION IN A QD WITH $N$ ODD}

\section{A. Energy structure}

When the number of electrons confined in the QD is odd and the magnetic field is weak enough, the ground and first excited states are usually the Zeeman $s_{z}=1 / 2$ and $s_{z}=-1 / 2$ sublevels of a doublet (Fig. 1). Since the initial and final spin states belong to the same orbital, $\Delta M=0$ and SO mixing (which requires $\Delta M= \pm 1$ ) is only possible with higher-lying states. In addition, the phonon energy (corresponding to the electron transition energy) is typically small (in the $\mu \mathrm{eV}$ scale). In this case, the relaxation rate is determined essentially by the phonon density, the strength and nature of the SO interaction, and the proximity of higher-lying states. ${ }^{9,11}$ In order to gain some insight on the influence of these factors, in Fig. 1 we compare the energy structure of a QD with $N=1,3,5$ vs an axial magnetic field, in the presence of Rashba and Dresselhaus interactions ${ }^{55}$ One can see that the increasing number of particles changes the energy magneto spectrum drastically. This is because the quantum numbers of the low-lying energy levels change, resulting in a different field dependence, and because Coulomb interaction leads to an increased density of electron states, as well as to a more complicated spectrum. 


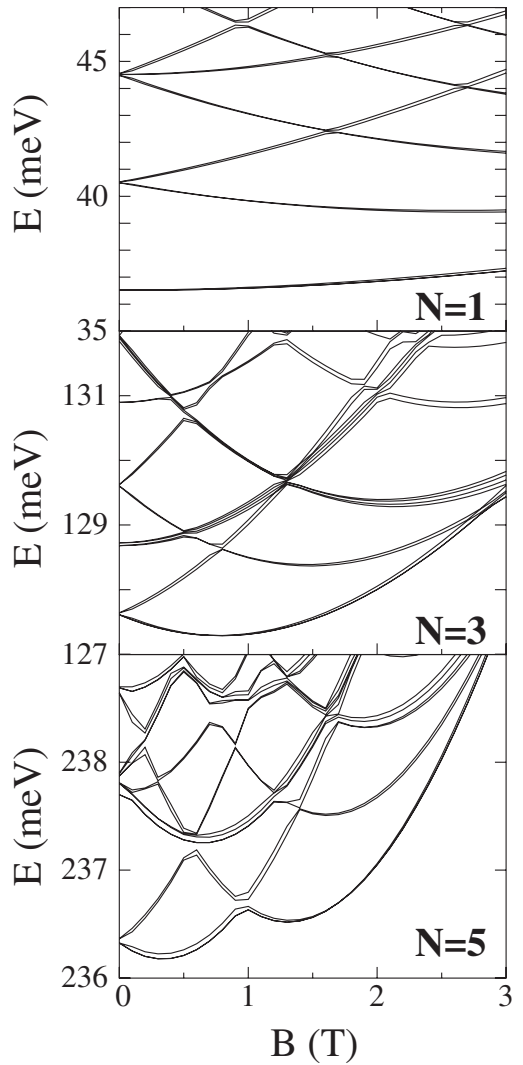

FIG. 1. Low-lying energy levels in a QD with $N=1,3,5$ interacting electrons, as a function of an axial magnetic field. The SO interaction coefficients are $\alpha=50 \mathrm{meV} \AA$ and $\beta=25 \mathrm{meV} \AA$. The dot has $\hbar \omega_{0}=4 \mathrm{meV}$ and $L_{z}=10 \mathrm{~nm}$. Note the increasing size of the SO-induced anticrossing gaps and zero-field splittings with increasing $N$.

At first sight, the energy spectra of Fig. 1 closely resemble those in the absence of SO effects. For instance, the $N=1$ spectrum is very similar to the pure Fock-Darwin spectrum. ${ }^{42}$ Rashba and Dresselhaus interactions were expected to split the degenerate $|m|>0$ shells at $B=0$, shift the positions of the level crossings and turn them into anticrossings, ${ }^{36,52-54}$ but here such signatures are hardly visible because SO interaction is weak in GaAs. In fact, the magnitude of the SO-induced zero-field energy splittings and that of the anticrossing gaps is of very few $\mu \mathrm{eV}$, and SO effects simply add fine features to the $N=1$ spectrum. ${ }^{52}$

A significantly different picture arises in the $N=3$ and $N$ $=5$ cases. Here, the increased density of electronic states enhances SO mixing as compared to the single-electron case. ${ }^{56}$ As a result, the anticrossing gaps can be as large as $30 \mu \mathrm{eV}(N=3)$ and $60 \mu \mathrm{eV}(N=5)$. Moreover, unlike in the $N=1$ case, where the ground state orbital has $m=0$, here it has $|M|=1$. Therefore, the Zeeman sublevels involved in the fundamental spin transition are subject to SO-induced zerofield splittings. To illustrate this point, in Fig. 2 we zoom in on the energy spectrum of the four lowest states of $N=3$ and $N=5$ under weak magnetic fields, without (left panels) and with (right panels) Rashba interaction. Clearly, the fourfold degeneracy of $|M|=1$ spin orbitals at $B=0$ has been lifted by SO interaction. ${ }^{36}$ One can also see that the order of the two

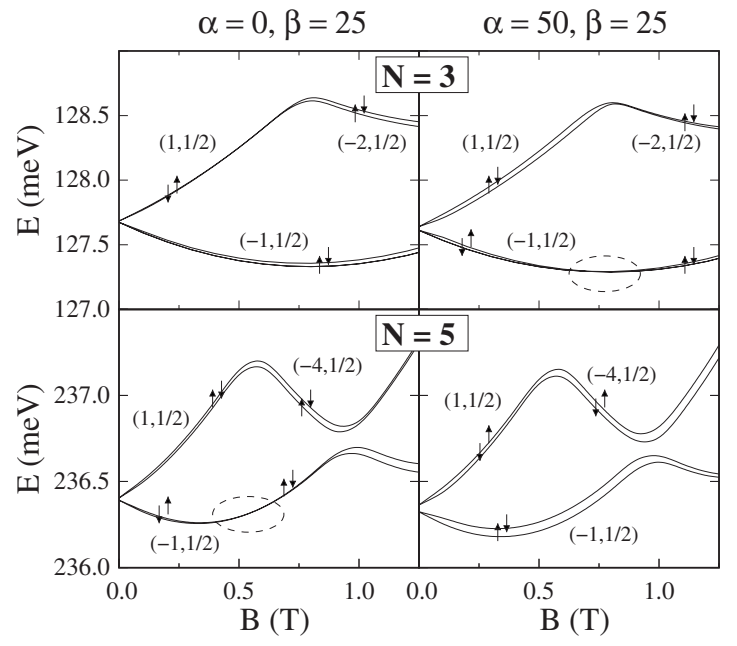

FIG. 2. The four lowest energy levels in a QD with $N=3,5$ interacting electrons, as a function of an axial magnetic field, without (left column) and with (right column) Rashba SO interaction. The approximate quantum numbers $(M, S)$ of the levels are shown, with arrows denoting the spin projection $S_{z}=1 / 2(\uparrow)$ and $S_{z}=-1 / 2$ $(\downarrow)$. The dashed circles highlight the region of intrinsic spin mixing of the ground state.

lowest sublevels at $B \sim 0$ changes when Rashba interaction is switched on. Thus, for $N=3$ and $\alpha=0$, the two lowest sublevels are $\left(M=-1, S_{z}=1 / 2\right)$ and $\left(M=-1, S_{z}=-1 / 2\right)$, but this order is reversed when $\alpha=50 \mathrm{meV} \AA$. The opposite level order as a function of $\alpha$ is found for $N=5$. This behavior constitutes a qualitative difference with respect to the $N=1$ case in two aspects. First, the phonon energy (i.e., the energy of the fundamental spin transition) is no longer given by the bare Zeeman splitting. Instead, it has a more complicated dependence on the magnetic field, and it is greatly influenced by the particular values of $\alpha$ and $\beta$. This is apparent in the $N=5$ panels, where the energy splitting between the two lowest states strongly differs depending on the relative value of $\alpha$ and $\beta$. Second, it is possible to find situations where the ground state at $B \sim 0$ has $S_{z}=-1 / 2$ and the first excited state has $S_{z}=1 / 2$ (e.g., $N=3$ when $\alpha>\beta$ or $N=5$ when $\alpha<\beta$ ). In these cases, the Zeeman splitting leads to a weak anticrossing of the two sublevels (highlighted with dashed circles in Fig. 2) which has no counterpart in single-electron systems. This kind of $B$-induced (i.e., not phonon-induced) ground state spin mixing, also referred to as "intrinsic spin mixing," has been previously reported for singlet-triplet transitions in $N=2$ QDs. ${ }^{58}$ Here we show that they may also exist in fewelectron QDs with $N$ odd.

Figure 1 puts forward yet another qualitative difference between SO coupling in single- and few-electron QDs: while in the former low-energy anticrossings are due to Rashba interaction, ${ }^{11,36,52}$ in few-electron QDs, when $S=3 / 2$ states come into play, both Rashba and Dresselhaus terms may induce anticrossings. For example, the $\left(M=-1, S_{z}=1 / 2\right)$ sublevel couples directly to both $\left(M=-2, S_{z}=-1 / 2\right)$ and $(M=$ $\left.-2, S_{z}=3 / 2\right)$ sublevels, via the Dresselhaus and Rashba interaction, respectively. Coupling to $S=3 / 2$ states is a characteristic feature of $N>1$ systems, which has important effects on the spin relaxation rate, as we will discuss below. 


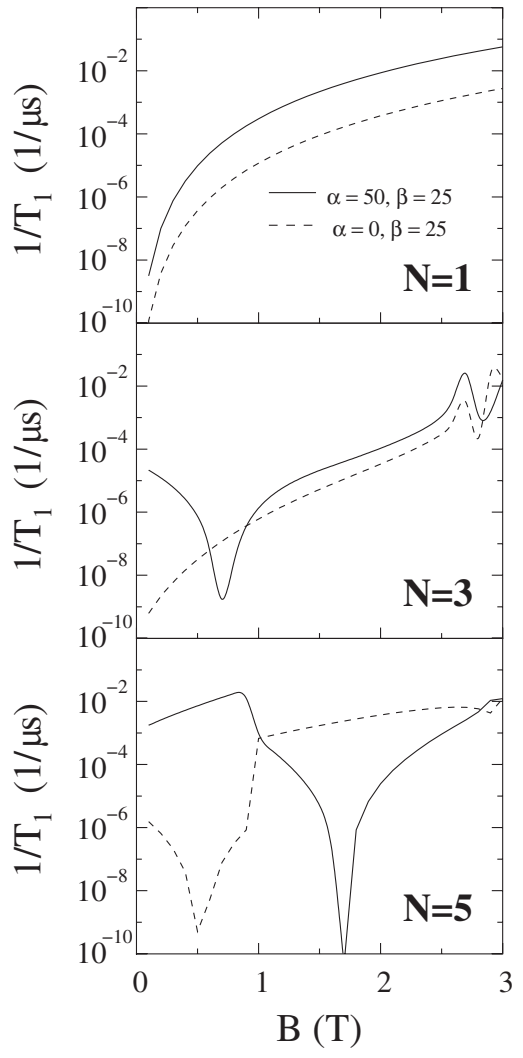

FIG. 3. Spin relaxation rate in a QD with $N=1,3,5$ interacting electrons as a function of an axial magnetic field. Solid (dashed) lines stand for the system with (without) Rashba interaction. Note the strong influence of the SO interaction in the shape of the relaxation curve for $N>1$.

\section{B. Spin relaxation between Zeeman sublevels}

In Fig. 3 we compare the magnetic field dependence of the spin relaxation rate between the two lowest Zeeman sublevels of $N=1,3,5$. Dashed lines (solid lines) are used for systems without (with) Rashba interaction. ${ }^{59}$ While for $N$ $=1$ the well-known exponential dependence with $B$ is found, ${ }^{2,6,9}$ and the main effect of Rashba coupling is to shift the curve upwards (i.e., to accelerate the relaxation), for $N$ $=3$ and $N=5$ the relaxation rate exhibits complicated trends which strongly depend on the values of the SO coupling parameters.

To understand this result, one has to bear in mind that in spin relaxation processes two well-distinguished and complementary ingredients are involved, namely SO interaction and phonon emission. Phonon emission grants the conservation of energy in the electron relaxation, but phonons have zero spin and therefore cannot couple states with different spin. It is the SO interaction that turns pure spin states into mixed ones, thus enabling the phonon-induced transition. The overall efficiency of the scattering event is then given by the combination of the two phenomena: the phonon emission efficiency modulated by the extent of the SO mixing. The shape of spin relaxation curves shown in Fig. 3 can be directly related to the energy dispersion of the phonon, which corresponds to the splitting between the two lowest levels of the electron spectrum. Thus, for $N=1$, the phonon

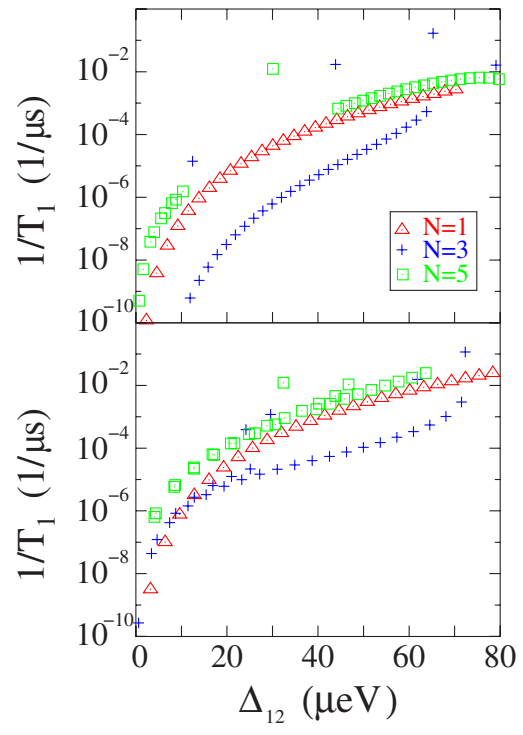

FIG. 4. (Color online) Spin relaxation rate in a QD with $N$ $=1,3,5$ interacting electrons as a function of the energy splitting between the two lowest spin states. Top panel: $\alpha=0, \beta$ $=25 \mathrm{meV} \AA$. Bottom panel: $\alpha=50 \mathrm{meV} \AA, \beta=25 \mathrm{meV} \AA$. The relaxation of $N=3$ is slower than that of $N=1$ for a wide range of $\Delta_{12}$. The irregular data distribution is due to the irregular relaxation rates vs magnetic field. For example, the strongly deviated points of $N$ $=3$ come from the peaks at $B \sim 3$ in Fig. 3 .

energy is simply proportional to $B$ through the Zeeman splitting, but for $N=3$ and $N=5$ it has a nontrivial dependence on $B$, as shown in Fig. 2. Actually, the relaxation minima in Fig. 3 are connected with the magnetic field values where the two lowest levels anticross in Fig. 2. In these magnetic field windows, in spite of the fact that SO coupling is strong, the phonon density is so small that the relaxation rate is greatly suppressed. ${ }^{28}$ Similarly, the relaxation rate fluctuations of $N$ $=3$ at $B \sim 3 \mathrm{~T}$ are signatures of the anticrossings with highangular momentum states. For larger fields $(B>3 \mathrm{~T})$, the ground state approaches the maximum density droplet configuration and high-spin states are possible. ${ }^{44}$ In this work, however, we restrict ourselves to the magnetic field regime where the ground state is a doublet.

For a more direct comparison between the relaxation rates of $N=1,3,5$, in Fig. 4 we replot the data of Fig. 3 as a function of the energy splitting between the two lowest states, $\Delta_{12}$, without (top panel) and with (bottom panel) Rashba interaction. Since the phonon energy is identical for all points with the same $\Delta_{12}$, differences in the relaxation rate arise exclusively from the different strength of SO interaction. $\Delta_{12}$ is also a relevant parameter from the experimental point of view, since it is usually required that it be large enough for the states to be resolvable. In this sense, it is worth noting that, even if the interlevel splittings shown in Fig. 4 are fairly small, a number of experiments have successfully addressed this regime..$^{5,8,21}$

A most striking feature observed in the figure is that, for most values of $\Delta_{12}$, the $N=3$ relaxation rate is clearly slower than the $N=1$ one. Likewise, $N=5$ shows a similar (or slightly faster) relaxation rate than $N=1$. These are interesting results, for they suggest that improved spin stability may 
be achieved using few-electron QDs instead of the singleelectron ones typically employed up to date. ${ }^{8}$ At first sight the results are surprising, because the higher density of states in the few-electron systems implies smaller interlevel spacings, and hence stronger SO mixing, which should translate into enhanced relaxation. It then follows that another physical mechanism must be acting upon the few-electron systems, which reduces the transition probability between the initial and final spin states, and may even make it smaller than for $N=1$. Here we propose that such mechanism is the SO admixture with low-lying quadruplet $(S=3 / 2)$ states, which become available for $N>1$. By coupling to $S=3 / 2$ levels, the projection of the doublet $S_{z}=1 / 2$ levels onto $S_{z}$ $=-1 / 2$ ones is reduced, and this partly inhibitis the transition between the lowest doublet sublevels.

Let us explain this by comparing the spin transition for $N=1$ and $N=3$. For $N=1$, the spin configuration of the initial and final states, in the absence of SO coupling, is $\left|S_{z}=-1 / 2\right\rangle$ and $\left|S_{z}=+1 / 2\right\rangle$, respectively. The transition between these states is spin forbidden. However, when SO coupling is switched on, the two states become admixed with higher-lying $S=1 / 2$ states fulfilling the $\Delta S_{z}= \pm 1$ condition. The transition between the initial and final states can then be represented schematically as

$$
\begin{aligned}
c_{a}\left|S_{z}=-1 / 2\right\rangle+c_{b}\left|S_{z}=+1 / 2\right\rangle \Rightarrow \\
c_{r}\left|S_{z}=+1 / 2\right\rangle+c_{s}\left|S_{z}=-1 / 2\right\rangle,
\end{aligned}
$$

where $c_{i}$ are the admixture coefficients (in general $c_{a} \gg c_{b}$ and $c_{r} \gg c_{s}$ ). Clearly now both spin configurations of the initial state have a finite overlap with the final state, and so the transition is possible. Let us next consider the $N=3$ case. In the absence of SO coupling, the initial and final states are again the $S_{z}=-1 / 2$ and $S_{z}=+1 / 2$ doublets, respectively, and the transition is spin forbidden. When we switch on SO coupling, we note that the $\Delta S_{z}= \pm 1$ condition allows for mixing not only with $S_{z}= \pm 1 / 2$ states (either doublets or quadruplets), but also with $S_{z}= \pm 3 / 2$ quadruplets, so that the transition can be represented as

$$
\begin{aligned}
c_{a}\left|S_{z}=-1 / 2\right\rangle+c_{b}\left|S_{z}=+1 / 2\right\rangle+c_{c}\left|S_{z}=-3 / 2\right\rangle \Rightarrow \\
c_{r}\left|S_{z}=+1 / 2\right\rangle+c_{s}\left|S_{z}=-1 / 2\right\rangle+c_{t}\left|S_{z}=+3 / 2\right\rangle,
\end{aligned}
$$

where, in general, $c_{a} \gg c_{b}, c_{c}$, and $c_{r} \gg c_{s}, c_{t}$. In this case, $\mid S_{z}$ $=-3 / 2\rangle$ has no overlap with the final state configurations. Likewise, $\left|S_{z}=+3 / 2\right\rangle$ has no overlap with the initial state configurations. Therefore, these quadruplet configurations are inactive from the point of view of the transition, and the more important they are (i.e., the stronger the SO coupling with quadruplet states), the less likely the transition is.

To prove this argument quantitatively, in Fig. 5 we illustrate the spin relaxation of $N=3$ calculated by diagonalization of the SO Hamiltonian including and excluding the lowlying $S=3 / 2$ states from the basis set. As expected, when the quadruplets are not considered, the transition is visibly faster. For $N=5$, low-lying $S=3 / 2$ levels are also available, but in this case they barely compensate for the large density of electron states, so that the overall scattering rate turns out to be comparable to that of $N=1$.

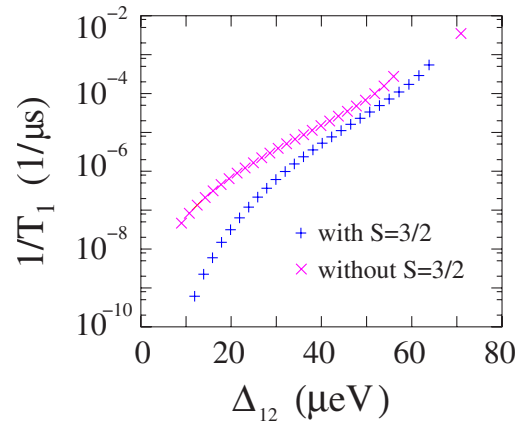

FIG. 5. (Color online) Spin relaxation rate in a QD with $N=3$ interacting electrons as a function of the energy splitting between the two lowest spin states. $\alpha=0$ and $\beta=25 \mathrm{meV} \AA$. Symbol $+(\times)$ stands for SO Hamiltonian diagonalized in a basis which includes (excludes) $S=3 / 2$ states. Clearly, the inclusion of $S=3 / 2$ states slows down the relaxation.

To test the robustness of the few-electron spin states stability predicted above, we also compare the relaxation rate of $N=1$ and $N=3$ in a QD with different confinement, namely $\hbar \omega_{0}=6 \mathrm{meV}$, in Fig. 6. Since the lateral confinement of the dot is now stronger, $(M=-1, S=1 / 2)$ is the $N=3$ ground state up to large values of the magnetic field $(B \sim 5 \mathrm{~T})$. This allows us to investigate larger Zeeman splittings (i.e., larger $\Delta_{12}$ ), which may be easier to resolve experimentally. As seen in the figure, the relaxation rate of $N=3$ is again slower than that of $N=1$ for a wide range of $\Delta_{12}$, the behavior being very similar to that of Fig. 4, albeit extended towards larger interlevel spacings. The crossing between $N=3$ and $N=1$ relaxation rates at large $\Delta_{12}$ values, both in Fig. 4 and Fig. 6, is due to the proximity of high-angular momentum levels coming down in energy for $N=3$ when the magnetic field (and

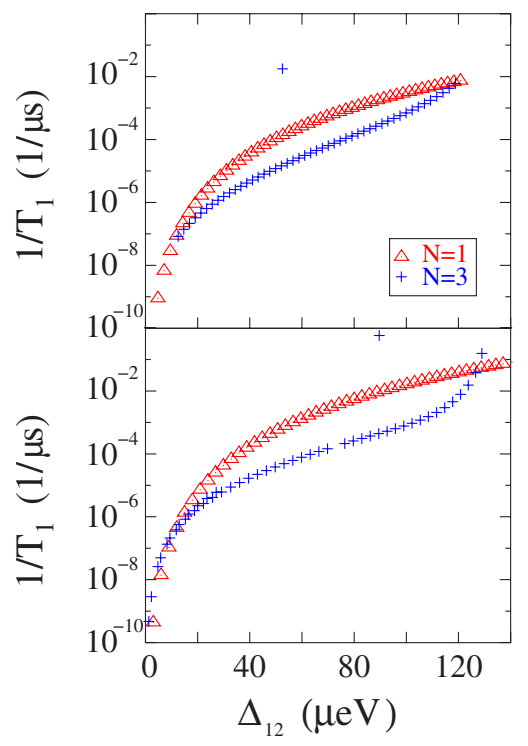

FIG. 6. (Color online) Spin relaxation rate in a QD with $N$ $=1,3$ interacting electrons as a function of the energy splitting between the two lowest spin states. The QD has $\hbar \omega_{0}=6 \mathrm{meV}$. Top panel: $\alpha=0, \quad \beta=25 \mathrm{meV} \AA$. Bottom panel: $\alpha=50 \mathrm{meV} \AA, \beta$ $=25 \mathrm{meV} \AA$. As for the weaker-confined dot of Fig. 4, the relaxation of $N=3$ is slower than that of $N=1$ for a wide range of $\Delta_{12}$. 


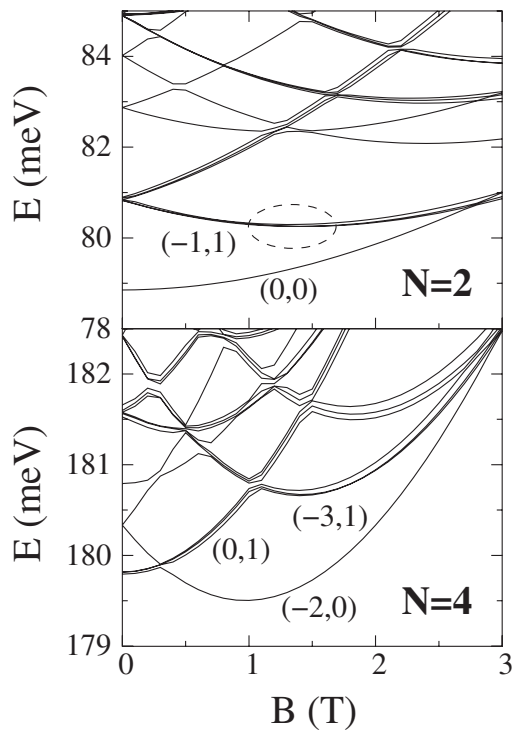

FIG. 7. Low-lying energy levels in a QD with $N=2,4$ interacting electrons as a function of an axial magnetic field. $\alpha$ $=50 \mathrm{meV} \AA$ and $\beta=25 \mathrm{meV} \AA$. The approximate quantum numbers $(M, S)$ of the lowest states are shown. The dashed circle in $N=2$ highlights the anticrossing between $M=-1$ Zeeman sublevels.

hence the Zeeman splitting) is large. Such levels bring about strong SO admixture and thus fast relaxation (see middle panel of Fig. 3 at $B \sim 3 \mathrm{~T}$ ).

\section{SPIN RELAXATION IN A QD WITH $N$ EVEN}

\section{A. Energy structure}

When the number of electrons confined in the QD is even and the magnetic field is not very strong, the ground and first excited states are usually a singlet $(S=0)$ and a triplet $(S$ $=1$ ) with three Zeeman sublevels $\left(S_{z}=+1,0,-1\right)$. Unlike in the previous section, here the initial and final states of the spin transition may have different orbital quantum numbers, and the interlevel splitting $\Delta_{12}$ may be significantly larger (in the meV scale). Under these conditions, the phonon emission efficiency no longer exhibits a simple proportionality with the phonon density, but it further depends on the ratio between the phonon wavelength and the QD dimensions. ${ }^{50,51}$ Moreover, SO interaction is sensitive to the quantum numbers of the initial and final electron states. ${ }^{26,28}$ Therefore, in this class of spin transitions the details of the energy structure are also relevant to determine the relaxation rate.

In Fig. 7 we plot the energy levels vs magnetic field for a QD with $N=2,4$ in the presence of Rashba and Dresselhaus interactions. The approximate quantum numbers $(M, S)$ of the lowest-lying states are written between parenthesis. For $N=2$ and weak fields, the ground state is the $(M=0, S=0)$ singlet, and the first excited state is the $(M=-1, S=1)$ triplet. As in the previous section, SO interaction introduces small zero-field splittings and anticrossings in the energy levels with $|M|>0 .{ }^{36}$ As a consequence, when $\alpha>\beta$, the zero-field ordering of the $(M=-1, S=1)$ Zeeman sublevels is such that they anticross in the presence of an external magnetic field.
This anticrossing is highlighted in the figure by a dashed circle. On the other hand, as $B$ increases the singlet-triplet energy spacing is gradually reduced, and then the singlet experiences a series of weak anticrossings with all three Zeeman sublevels of the triplet. These anticrossings are due to the fact that $\left(M=0, S=0, S_{z}=0\right)$ couples to the $(M=-1, S$ $\left.=1, S_{z}=-1\right)$ sublevel via Dresselhaus interaction, to the $(M$ $\left.=-1, S=1, S_{z}=+1\right)$ sublevel via Rashba interaction, and finally to the $\left(M=-1, S=1, S_{z}=0\right)$ sublevel indirectly through higher-lying states. ${ }^{26,28}$

For $N=4$, the density of electronic states is larger than for $N=2$, which again reflects in a larger magnitude of the anticrossings gaps due to the enhanced SO interaction. The ground state at $B=0$ is a triplet $(M=0, S=1)$, but soon after it anticrosses with a singlet $(M=-2, S=0)$. After this, and before the formation of Landau levels, two different branches of the first excited state can be distinguished: when $B<1 \mathrm{~T}$, the first excited state is $(M=0, S=1)$, and when $B$ $>1 \mathrm{~T}$ it is $(M=-3, S=1)$. It is worth pointing out that the complexity of the $N=4$ spectrum, as compared to the simple $N=2$ one, implies a greater flexibility to select initial and final spin states by means of external fields. As we shall discuss below, this degree of freedom has important consequences on the relaxation rate.

\section{B. Triplet-singlet spin relaxation}

In a recent work, we have investigated the magnetic field dependence of the TS relaxation due to SO coupling and phonon emission in $N=2$ and $N=4$ QDs. ${ }^{28}$ Here we study this kind of transition from a different perspective, namely we compare the spin relaxation of two- and four-electron systems in order to highlight the changes introduced by interelectron repulsion. Increasing the number of electrons confined in the QD has three important consequences on the TS transition. First, it increases the density of electronic states (and then the SO mixing), leading to faster relaxation. Second, as mentioned in the previous section, it introduces a wider choice of orbital quantum numbers for the singlet and triplet states. Third, it increases the strength of electronic correlations. Since now the initial and final spin states have different orbital wave functions, the latter factor effectively reduces phonon scattering, in a similar fashion to charge relaxation processes ${ }^{33}$ (this effect has been recently pointed out in Ref. 30 as well). To find out the overall combined effect of these three factors, in this section we analyze quantitative simulations of correlated QDs.

We focus on the magnetic field regions where the ground state is a singlet and the excited state is a triplet. A complete description of the TS transition should then include spin relaxation between the Zeeman-split sublevels of the triplet. However, for the weak fields we consider this relaxation is orders of magnitude slower than the TS one (compare Figs. 3 and 8), ${ }^{60}$ the reason for this being the small Zeeman energy and the fact that the Zeeman sublevels are not directly coupled by Rashba and Dresselhaus terms, as mentioned in Sec. III. Therefore, it is a good approximation to assume that all three triplet Zeeman sublevels are equally populated and they relax directly to the singlet. ${ }^{26}$ 


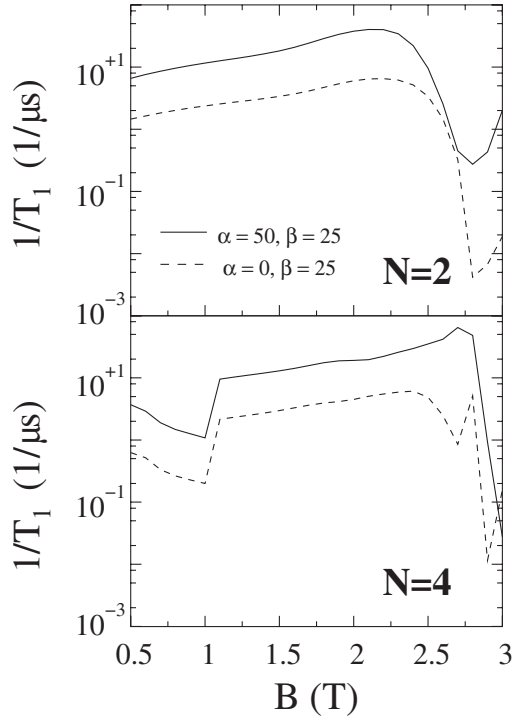

FIG. 8. Spin relaxation rate in a QD with $N=2,4$ interacting electrons as a function of an axial magnetic field. Solid (dashed) lines stand for the system with (without) Rashba interaction. The relaxation of $N=4$ when $B<1 \mathrm{~T}$ is slower than that of $N=2$.

Figure 8 represents the TS relaxation rate in a QD with $N=2,4$, after averaging the relaxation from the three triplet sublevels. Solid (dashed) lines stand for the case with (without) Rashba interaction. ${ }^{59}$ The main effect of Rashba and Dresselhaus interactions is to accelerate the spin transition by shifting the relaxation curve upwards. This is in contrast to the $N$-odd case, where these terms may induce drastic changes in the shape of the relaxation rate curve (see Fig. 3). Figure 8 also reveals a different behavior of the $N=2$ and $N=4$ TS relaxation rates. The former increases gradually with $B$ and then drops in the vicinity of the TS anticrossing, due to the small phonon energies. ${ }^{28-30}$ Conversely, for $N=4$ an additional feature is found, namely an abrupt step at $B$ $\sim 1$. This is due to the change of angular momentum of the excited triplet. For $B<1 \mathrm{~T}$ the triplet has $M=0$, and for $B$ $>1 \mathrm{~T}$ it has $M=-3$. Since the ground state is a singlet with $M=-2$, the $M=0$ triplet does not fulfill the $\Delta M= \pm 1$ condition for linear SO coupling. This inhibits direct spin mixing between initial and final states and reduces the relaxation rate by about one order of magnitude. ${ }^{28}$

Noteworthy, the choice of states differing in more than one quantum of angular momentum is only possible for $N$ $>2$ QDs. One may then wonder if it is more convenient to use these systems instead of the $N=2$ ones dominating the experimental literature up to date, ${ }^{20,21,29}$ i.e., if it compensates for the increased density of electronic states. Interestingly, Fig. 8 predicts slower relaxation for the $N=4$ QD with $M=0$ triplet than for $N=2$. To verify that this arises from weakend SO coupling rather than from different phonon energy values, in Fig. 9 we replot the spin relaxation rate of $N=2,4$ as a function of the TS energy splitting. In the figure, the upper and bottom panels represent the situations without and with Rashba interaction, respectively. While $N=4$ shows similar relaxation rate to $N=2$ when the triplet has $M=-3$, the relaxation is slower by about one order of magnitude when the triplet has $M=0$. This result indicates that the

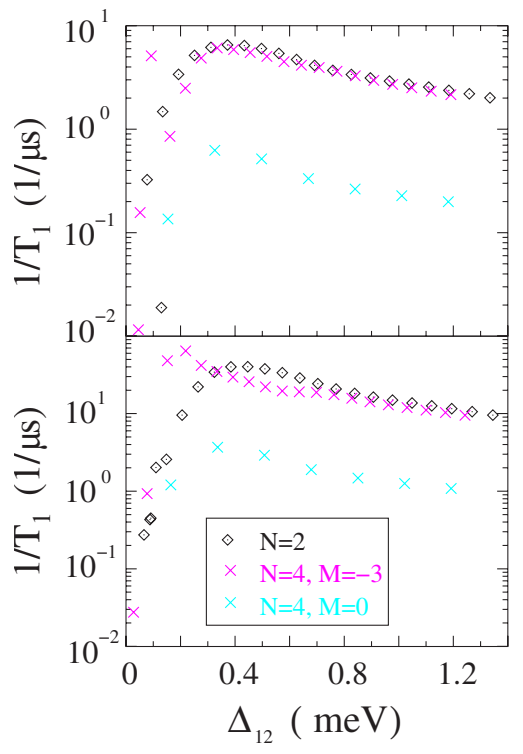

FIG. 9. (Color online) Spin relaxation rate in a QD with $N$ $=2,4$ interacting electrons as a function of the energy spacing between the singlet and the triplet. Here $M$ stands for the angular momentum of the triplet. Top panel: $\alpha=0, \beta=25 \mathrm{meV} \AA$. Bottom panel: $\alpha=50 \mathrm{meV} \AA, \beta=25 \mathrm{meV} \AA$. The relaxation of $N=4$ is comparable to that of $N=2$ when the triplet has $M=-3$, and it is much smaller when $M=0$.

weakening of SO mixing due to the violation of the $\Delta M$ $= \pm 1$ condition clearly exceeds the strengthening due to the higher density of states, confirming that $N=4$ systems are more attractive than $N=2$ ones to obtain long triplet lifetimes. We also point out that, in spite of the different density of states, the relaxation rate of $N=2$ and $N=4, M=-3$ triplets is quite similar. This can be ascribed to the phonon scattering reduction by electronic correlations, ${ }^{33}$ which may also explain the fact that experimentally resolved TS relaxation rates of $N=8$ QDs and $N=2$ QDs be quite similar. ${ }^{20,31}$

\section{COMPARISON WITH $N=2$ EXPERIMENTS}

Whereas, to our knowledge, no experiments have measured transitions between Zeeman-split sublevels in $N>1$ systems yet, a number of works have dealt with TS relaxation in QDs with few interacting electrons. In Ref. 28 we showed that our model correctly predicts the trends observed in experiments with $N=2$ and $N=8$ QDs subject to axial magnetic fields. ${ }^{20,21,31}$ In this section, we extend the comparison to experiments available for $N=2$ TS relaxation in QDs, ${ }^{29}$ which provide continuous measurements of the average triplet lifetime against axial magnetic fields, from $B=0$ to the vicinity of the TS anticrossing. By using a simple model, the authors of the experimental work showed that the measurements are in clear agreement with the behavior expected from SO coupling plus acoustic phonon scattering. However, in such model: (i) the TS energy splitting was a taken directly from the experimental data, (ii) the SO coupling effect was accounted for by parametrizing the admixture of the lowest singlet and triplet states only, and (iii) the $B$ dependence of the SO-induced admixture was neglected. 


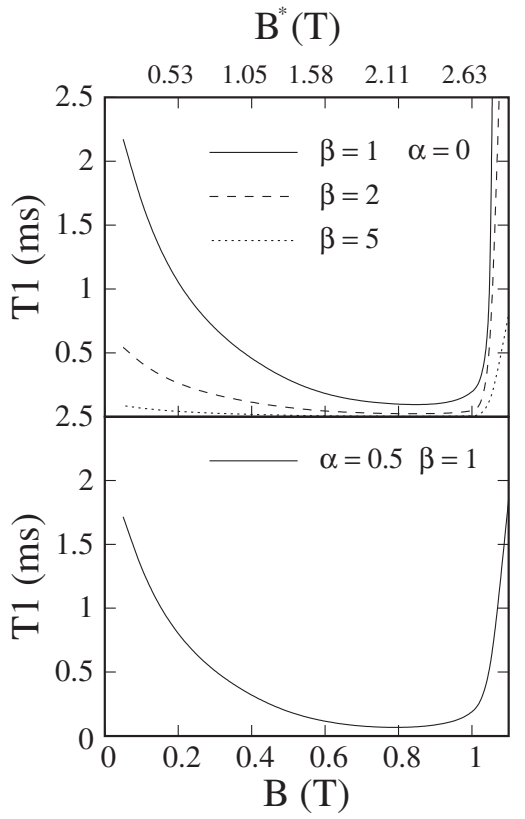

FIG. 10. Average triplet lifetime in a QD with $N=2$ electrons as a function of an axial magnetic field. Only the field region before the TS anticrossing is shown. $\alpha$ and $\beta$ are in meV $\AA$ units. $B$ is the applied axial magnetic field, and $B^{*}$ is the equivalent tilted magnetic field, for comparison with Ref. 29 experiment.

Approximation (ii) may overlook the correlation-induced reduction of phonon scattering ${ }^{30,33}$ that we have shown above to be significant, and which may have an important contribution from higher excited states in weakly confined QDs. In turn, approximation (iii) may overlook the important influence of SO coupling in the $B$ dependence of the triplet lifetime, as we had anticipated in Ref. 28. Here we compare with the experimental findings using our model, which includes these effects properly. We assume a QD with an effective well width $L_{z}=30 \mathrm{~nm}$, as expected by Ref. 29 authors, and a lateral confinement parabola of $\hbar \omega_{0}=2 \mathrm{meV}$ which, as we shall see next, fits well the position of the TS anticrossing. Yet, the comparison is limited by the lack of detailed information about the Rashba and Dresselhaus interaction constants, and because we deal with circular QDs instead of elliptical ones (the latter effect introduces simple deviations from the circular case $\left.{ }^{26}\right)$. In addition, in the experiment a tilted magnetic field of magnitude $B^{*}$, forming an angle of $68^{\circ}$ with the vertical direction was used. Here we consider the vertical component of the field $\left(B=0.37 B^{*}\right)$, which is the main responsible for the changes in the energy structure, and the effect of the in-plane component enters via the Zeeman splitting only.

Figure 10 illustrates the average triplet lifetime for $N=2$. The bottom axis shows the vertical magnetic field $B$ value, while the top axis shows the value to be compared with the experiment $B^{*}{ }^{59}$ As can be seen, the triplet lifetime first decreases with the field and then it abruptly increases in the vicinity of the TS anticrossing, due to the small phonon density. ${ }^{28}$ This behavior is in clear agreement with the experiment (cf. Fig. 3 of Ref. 29). The position of the anticrossing $\left(B^{*} \sim 2.9 \mathrm{~T}\right)$ is also close to the experimental value $\left(B^{*}\right.$ $\sim 2.8 \mathrm{~T}$ ), which confirms that that $\hbar \omega_{0}=2 \mathrm{meV}$ is similar to the mean confinement frequency of the experimental sample. A departure from the experimental trend appears at weak fields $(B<0.5 \mathrm{~T})$, where we observe a continuous increase of $T_{1}$ with decreasing $B$, while the experiment reports a plateau. This is most likely due to the ellipticity of the experimental sample, which renders the electron states (and consequently the relaxation rate) insensitive to the field in the $B^{*}$ $=0-0.5 \mathrm{~T}$ region (see Fig. $1 \mathrm{a}$ in 29 ). In any case, Fig. 10 clearly confirms the role of phonon-induced relaxation in the experiments, using a realistic model for the description of correlated electron states, SO admixture, and phonon scattering.

A comment is worth it here on the magnitude of the SO coupling terms. In Fig. 10, we obtain good agreement with the experimental relaxation times by using small values of the SO coupling parameters. In particular, a close fit is obtained using $\beta=1, \alpha=0.5 \mathrm{meV} \AA$, which yields a spin-orbit length $\lambda_{S O}=48 \mu \mathrm{m}$. This value, which coincides with the experimental guess $\left(\lambda_{S O} \approx 50 \mu \mathrm{m}\right)$, indicates that SO coupling is several times weaker than that reported for other GaAs QDs. ${ }^{8}$ Typical GaAs parameters are often larger. For instance, measurements of the Rashba and Dresselhaus constants by analysis of the weak antilocalization in clean GaAs/AlGaAs two-dimensional gases revealed $\alpha$ $=4-5 \mathrm{meV} \AA$, and $\gamma_{c}=28 \mathrm{eV} \AA^{3}$ (i.e., $\beta=3 \mathrm{meV} \AA$ for our quantum well of $\left.L_{z}=30 \mathrm{~nm}\right){ }^{61}$ To be sure, the small SO coupling parameters in the experiment have a major influence on the lifetime scale. Compare, e.g., the $\beta=1$ and $\beta=5 \mathrm{meV} \AA$ curves in Fig. 10. Actually, we note that accurate comparison with the timescale reported for other GaAs samples ${ }^{31}$ is also possible within our model, but assuming stronger SO coupling constants. ${ }^{28}$ In Ref. 29 , it was suspected that the weak SO coupling inferred from the experimental data could be the result of the exclusion of higher orbitals and the magnetic field dependence of SO admixture in their model (higher states reduce the effective SO coupling constants by decreasing the phonon-induced scattering ${ }^{30,33}$ ). Here we have considered both these effects and still small SO coupling constants are needed to reproduce the experiment. Therefore, understanding the origin of their small value remains as an open question. One possibility could be that the particular direction of the tilted magnetic field used in the experiment corresponded to a reduced degree of SO admixture. ${ }^{30}$

\section{CONCLUSIONS}

We have investigated theoretically the energy structure and spin relaxation rate of weakly confined QDs with $N$ $=1-5$ interacting electrons, subject to axial magnetic fields, in the presence of linear Rashba and Dresselhaus SO interactions. It has been shown that the number of electrons confined in the dot introduces changes in the energy spectrum which significantly influence the intensity of the SO admixture, and hence the spin relaxation. In general, the larger the number of confined carriers, the higher the density of electronic states. This decreases the energy splitting between consecutive levels and then enhances SO admixture, which should lead to faster spin relaxation. However, we find that 
this is not necessarily the case, and slower relaxation rate may be found for few-electron QDs as compared to the usual single- and two-electron QDs used up to date. The physical mechanisms responsible for this have been identified. For $\mathrm{N}$-odd systems, when the spin transition takes place between Zeeman-split sublevels, it is the presence of low-energy $S$ $=3 / 2$ states for $N>1$ that reduces the projection of the doublet $S_{z}=1 / 2$ sublevels into $S_{z}=-1 / 2$ ones, thus partly inhibiting the spin transition. For $N$-even systems, when the spin transition takes place between triplet and singlet levels, there are two underlying mechanisms. On the one hand, electronic correlations tend to reduce phonon emission efficiency. On the other hand, for $N>2$ a magnetic field can be used to select a pair of singlet-triplet states which do not fulfill the $\Delta M= \pm 1$ condition of direct $\mathrm{SO}$ admixture, which significantly weakens the SO mixing.
Last, we have compared our estimates with recent experimental data for TS relaxation in $N=2$ QDs. $^{29}$ Our results support the interpretation of the experiment in terms of $\mathrm{SO}$ admixture plus acoustic phonon scattering, even though quantitative agreement with the experiment requires assuming much weaker SO coupling than that reported for similar GaAs structures.

\section{ACKNOWLEDGMENTS}

We acknowledge support from the Italian Ministry for University and Scientific Research under FIRB Contract No. RBIN04EY74, Cineca Calcolo parallelo 2006, and Marie Curie IEF Project No. NANO-CORR MEIF-CT-2006023797.
*URL: www.nanoscience.unimore.it; climente@ unimore.it

${ }^{1}$ I. Zutic, J. Fabian, and S. Das Sarma, Rev. Mod. Phys. 76, 323 (2004).

${ }^{2}$ D. Heiss, M. Kroutvar, J. J. Finley, and G. Abstreiter, Solid State Commun. 135, 519 (2005).

${ }^{3}$ D. Loss and D. P. DiVincenzo, Phys. Rev. A 57, 120 (1998).

${ }^{4}$ V. N. Golovach, A. Khaetskii, and D. Loss, Phys. Rev. Lett. 93, 016601 (2004).

${ }^{5}$ R. Hanson, B. Witkamp, L. M. K. Vandersypen, L. H. Willems van Beveren, J. M. Elzerman, and L. P. Kouwenhoven, Phys. Rev. Lett. 91, 196802 (2003).

${ }^{6}$ M. Kroutvar, Y. Ducommun, D. Heiss, M. Bichler, D. Schuh, G. Abstreiter, and J. J. Finley, Nature (London) 432, 81 (2004).

${ }^{7}$ J. M. Elzerman, R. Hanson, L. H. Willems van Beveren, B. Witkamp, L. M. K. Vandersypen, and L. P. Kouwenhoven, Nature (London) 430, 431 (2004).

${ }^{8}$ S. Amasha, K. MacLean, I. Radu, D. M. Zumbühl, M. A. Kastner, M. P. Hanson, and A. C. Gossard, arXiv:cond-mat/0607110.

${ }^{9}$ A. V. Khaetskii and Y. V. Nazarov, Phys. Rev. B 64, 125316 (2001).

${ }^{10}$ J. L. Cheng, M. W. Wu, and C. Lü, Phys. Rev. B 69, 115318 (2004).

${ }^{11}$ D. V. Bulaev and D. Loss, Phys. Rev. B 71, 205324 (2005).

${ }^{12}$ C. F. Destefani and S. E. Ulloa, Phys. Rev. B 72, 115326 (2005).

${ }^{13}$ P. Stano and J. Fabian, Phys. Rev. B 74, 045320 (2006).

${ }^{14}$ Y. Y. Wang and M. W. Wu, Phys. Rev. B 74, 165312 (2006).

${ }^{15}$ E. Ya. Sherman and D. J. Lockwood, Phys. Rev. B 72, 125340 (2005).

${ }^{16}$ L. M. Woods, T. L. Reinecke, and Y. Lyanda-Geller, Phys. Rev. B 66, 161318(R) (2002).

${ }^{17}$ I. A. Merkulov, Al. L. Efros, and M. Rosen, Phys. Rev. B 65, 205309 (2002).

${ }^{18}$ S. I. Erlingsson and Y. V. Nazarov, Phys. Rev. B 66, 155327 (2002).

${ }^{19}$ P. San-Jose, G. Zarand, A. Shnirman, and G. Schön, Phys. Rev. Lett. 97, 076803 (2006).

${ }^{20}$ T. Fujisawa, D. G. Austing, Y. Tokura, Y. Hirayama, and S. Tarucha, Nature (London) 419, 278 (2002); J. Phys.: Condens. Matter 15, R1395 (2003).
${ }^{21}$ R. Hanson, L. H. Willems van Beveren, I. T. Vink, J. M. Elzerman, W. J. M. Naber, F. H. L. Koppens, L. P. Kouwenhoven, and L. M. K. Vandersypen, Phys. Rev. Lett. 94, 196802 (2005).

${ }^{22}$ J. R. Petta, A. C. Johnson, J. M. Taylor, E. A. Laird, A. Yacoby, M. D. Lukin, C. M. Marcus, M. P. Hanson, and A. C. Gossard, Science 309, 2180 (2005).

${ }^{23}$ A. C. Johnson, J. R. Petta, J. M. Taylor, A. Yacoby, M. D. Lukin, C. M. Marcus, M. P. Hanson, and A. C. Gossard, Nature (London) 435, 925 (2005).

${ }^{24}$ J. R. Petta, A. C. Johnson, A. Yacoby, C. M. Marcus, M. P. Hanson, and A. C. Gossard, Phys. Rev. B 72, 161301(R) (2005).

${ }^{25}$ W. A. Coish and D. Loss, Phys. Rev. B 72, 125337 (2005).

${ }^{26}$ M. Florescu, S. Dickman, M. Ciorga, A. Sachrajda, and P. Hawrylak, Physica E (Amsterdam) 22, 414 (2004); M. Florescu and P. Hawrylak, Phys. Rev. B 73, 045304 (2006).

${ }^{27}$ D. Chaney and P. A. Maksym, Phys. Rev. B 75, 035323 (2007).

${ }^{28}$ J. I. Climente, A. Bertoni, G. Goldoni, M. Rontani, and E. Molinari, Phys. Rev. B 75, 081303(R) (2007).

${ }^{29}$ T. Meunier, I. T. Vink, L. H. Willems van Beveren, K. J. Tielrooij, R. Hanson, F. H. L. Koppens, H. P. Tranitz, W. Wegscheider, L. P. Kouwenhoven, and L. M. K. Vandersypen, Phys. Rev. Lett. 98, 126601 (2007).

${ }^{30}$ V. N. Golovach, A. Khaetskii, and D. Loss, arXiv:cond-mat/ 0703427.

${ }^{31}$ S. Sasaki, T. Fujisawa, T. Hayashi, and Y. Hirayama, Phys. Rev. Lett. 95, 056803 (2005).

${ }^{32}$ M. Ciorga, A. S. Sachrajda, P. Hawrylak, C. Gould, P. Zawadzki, S. Jullian, Y. Feng, and Z. Wasilewski, Phys. Rev. B 61, R16315 (2000); H. Drexler, D. Leonard, W. Hansen, J. P. Kotthaus, and P. M. Petroff, Phys. Rev. Lett. 73, 2252 (1994).

${ }^{33}$ A. Bertoni, M. Rontani, G. Goldoni, and E. Molinari, Phys. Rev. Lett. 95, 066806 (2005); J. I. Climente, A. Bertoni, M. Rontani, G. Goldoni, and E. Molinari, Phys. Rev. B 74, 125303 (2006).

${ }^{34}$ T. Chakraborty and P. Pietiläinen, Phys. Rev. B 71, 113305 (2005).

${ }^{35}$ P. Pietiläinen and T. Chakraborty, Phys. Rev. B 73, 155315 (2006).

${ }^{36}$ C. F. Destefani, S. E. Ulloa, and G. E. Marques, Phys. Rev. B 70, 205315 (2004). 
${ }^{37}$ During the finalization of this paper we have learned about a parallel work investigating the influence of Coulomb interaction in two-electron TS relaxation (Ref. 30). Many of the findings in the paper are in agreement with our numerical results.

${ }^{38}$ Y. A. Bychkov and E. I. Rashba, J. Phys. C 17, 6039 (1984).

${ }^{39}$ G. Dresselhaus, Phys. Rev. 100, 580 (1955).

${ }^{40}$ M. Rontani, C. Cavazzoni, D. Bellucci, and G. Goldoni, J. Chem. Phys. 124, 124102 (2006).

${ }^{41} \mathrm{http}: / /$ www.s3.infm.it/donrodrigo

${ }^{42}$ L. Jacak, P. Hawrylak, and A. Wojs, Quantum Dots (SpringerVerlag, Berlin, 1998).

${ }^{43}$ M. Brasken, S. Corni, M. Lindberg, J. Olsen, and D. Sundholm, Mol. Phys. 100, 911 (2002).

${ }^{44}$ P. Lucignano, B. Jouault, and A. Tagliacozzo, Phys. Rev. B 69, 045314 (2004)

${ }^{45}$ The $\left(M, S, S_{z}\right)$ quantum numbers of few-electron states are a good approximation for the lowest-lying states only. For higher-lying states, the energy spectrum becomes denser and the SO interaction becomes very strong even for GaAs, which leads to important departures from the SO-free picture. This does not occur in single-electron parabolic QDs because the energy levels are equally spaced.

${ }^{46}$ The convenience of using exact diagonalization procedures, instead of perturbational approaches, to account for the SO coupling in GaAs QDs has been claimed in Ref. 10.

${ }^{47}$ Physics of Hot Electron Transport in Semiconductors, edited by C. S. Ting (World Scientific, Singapore, 1992).

${ }^{48}$ Numerical Data and Functional Relationships in Science and Technology, edited by O. Madelung, Landolt-Börnstein Vol. 17, (Springer-Verlag, Berlin, 1982).

${ }^{49}$ M. Cardona, N. E. Christensen, and G. Fasol, Phys. Rev. B 38, 1806 (1988).
${ }^{50}$ U. Bockelmann, Phys. Rev. B 50, 17271 (1994).

${ }^{51}$ J. I. Climente, A. Bertoni, G. Goldoni, and E. Molinari, Phys. Rev. B 74, 035313 (2006).

${ }^{52}$ P. Stano and J. Fabian, Phys. Rev. B 72, 155410 (2005).

${ }^{53}$ O. Voskoboynikov, C. P. Lee, and O. Tretyak, Phys. Rev. B 63, 165306 (2001).

${ }^{54}$ W. H. Kuan and C. S. Tang, J. Appl. Phys. 95, 6368 (2004).

${ }^{55}$ The energy magneto spectrum of GaAs parabolic QDs with SO interaction and up to four interacting electrons was also investigated in Ref. 35, but considering Rashba interaction only.

${ }^{56}$ Coulomb-enhanced SO interaction was previously predicted for higher-dimensional structures (Ref. 57). Here we report it for QDs.

${ }^{57}$ G. H. Chen and M. E. Raikh, Phys. Rev. B 60, 4826 (1999).

${ }^{58}$ C. F. Destefani, S. E. Ulloa, and G. E. Marques, Phys. Rev. B 69, 125302 (2004).

${ }^{59}$ For simplicity of the discussion, in Figs. 3, 8, and 10, the near vicinity of $B=0 \mathrm{~T}$ is not shown. In that range one finds damped phonon-induced relaxation rates due to degeneracies arising from the time-reversal symmetry and the circular symmetry of the confinement we have assumed. We do not expect these features to be observable in experiments, because QDs are not perfectly circular and because hyperfine interaction is expected to be the dominant spin relaxation mechanism for very weak fields (see Refs. 18 and 23).

${ }^{60}$ Greatly suppressed TS spin relaxation, comparable to that of inter-Zeeman sublevels at very weak $B$, may be achieved by means of geometrically or field-induced acoustic phonon emission minima (Refs. 27 and 28).

${ }^{61}$ J. B. Miller, D. M. Zumbühl, C. M. Marcus, Y. B. Lyanda-Geller, D. Goldhaber-Gordon, K. Campman, and A. C. Gossard, Phys. Rev. Lett. 90, 076807 (2003). 